\title{
Histopathological diagnoses on pleural biopsy specimens over a 15-year period at Chris Hani Baragwanath Academic Hospital, Johannesburg, South Africa: A retrospective review
}

\author{
J R Edgar, ${ }^{1} \mathrm{MB}$ BCh; M L Wong, ${ }^{2} \mathrm{MB}$ BCh, DCH (SA), FCP (SA), FCCP, FRCP (Lond); M Hale ${ }^{3}$ MB ChB, LRCP, LRCS (Glasg), \\ FC Path (SA), LRCP\&S (Edin); C N Menezes, ${ }^{4}$ MD, MMed (Int Med), Dip HIV Man (SA), DTM\&H, FCP (SA), Cert ID (SA), PhD \\ ${ }^{1}$ Department of Internal Medicine, Faculty of Health Sciences, University of the Witwatersrand, Johannesburg, South Africa \\ ${ }^{2}$ Division of Pulmonology, Department of Internal Medicine, Faculty of Health Sciences, University of the Witwatersrand and Chris Hani \\ Baragwanath Academic Hospital, Johannesburg, South Africa \\ ${ }^{3}$ Department of Anatomical Pathology, National Health Laboratory Service, Johannesburg, South Africa \\ ${ }^{4}$ Division of Infectious Diseases, Department of Internal Medicine, Faculty of Health Sciences, University of the Witwatersrand and Chris Hani \\ Baragwanath Academic Hospital, Johannesburg, South Africa
}

Correspondingauthor: J R Edgar (jasedgar@icloud.com)

\begin{abstract}
Background. Pleural effusions are a common reason for presentation to healthcare facilities. Blind closed pleural biopsy can be a useful tool to diagnose their cause, especially in resource-limited settings.

Objectives. To determine the aetiology, frequency and change in profile of histopathological diagnoses made at Chris Hani Baragwanath Academic Hospital (CHBAH), Johannesburg, South Africa, over the period 1 January 2001 - 31 December 2015.

Methods. Pleural biopsies performed at CHBAH and analysed by histopathologists from the National Health Laboratory Service at the hospital over the study period were retrospectively reviewed by accessing reports from two databases (DISA and TrakCare). The subjects' ages, genders, HIV status and histopathological diagnoses as well as adenosine deaminase and Ziehl-Neelsen results were recorded.

Results. A total of 1013 samples were included in the study, with 780 considered adequate for assessment. The most common diagnosis was granulomatous inflammation $(48.1 \%, n=375)$, with the most common type being necrotising granulomatous inflammation (73.6\%, $n=276)$. Ten percent of biopsies ( $n=78)$ showed malignancy, most commonly adenocarcinoma, with $46.2 \%(n=36)$ metastatic and $23.1 \%$ $(n=18)$ primary lung adenocarcinoma. The odds of being diagnosed with malignancy showed increasing statistical significance above the age of 40 years: 40 - 49 years odds ratio (OR) 8.7, 95\% confidence interval (CI) 1.1 - 66.9 ( $p=0.038$ ); 50 - 59 years OR $12.4,95 \%$ CI 1.6 - 95.0 $(p=0.015) ; \geq 60$ years OR 23.0, 95\% CI $3.1-171.3$ ( $p=0.002$ ). HIV seropositivity was associated with lower odds of being diagnosed with malignancy compared with HIV-negative patients (OR $0.5,95 \%$ CI $0.2-0.9 ; p=0.040$ ), with greater odds of a 'non-cancer' diagnosis in HIVpositive patients (including granulomatous inflammation and pleuritis (OR 2.16, 95\% CI $1.03-4.51 ; p=0.040$ )).

Conclusions. Blind closed pleural biopsy has a role to play in the diagnosis of exudative pleural effusions in resource-limited settings, particularly for patients suspected to have tuberculosis (TB) or malignancy. TB remains a common cause of exudative pleural effusions. Patients aged $>40$ years presenting with an exudative pleural effusion should routinely have pleural biopsy performed. However, this study showed a high frequency of inadequate specimens from closed pleural biopsy. Training in the performance of this procedure to increase diagnostic rates is recommended.
\end{abstract}

S Afr Med J 2019;109(1):53-57. DOI:10.7196/SAMJ.2019.v109i1.13400

Pleural effusions are a common reason for patients to present to healthcare facilities around the world. The diagnostic workup of exudative pleural effusions may require pleural biopsy for histopathological assessment in order to reach a diagnosis. ${ }^{[1]}$ Closed blind pleural biopsy is a relatively cost-effective investigation that can be used to diagnose the cause of an exudative pleural effusion. The term 'blind' in this setting refers to obtaining tissue from the parietal pleura without directly visualising the area being sampled.

The predominant diagnoses after closed pleural biopsy in other studies included tuberculosis (TB) and malignancy. The prevalence of $\mathrm{TB}$ across studies varies, with rates up to $64.8 \%^{[2-6]}$ depending on the population studied, rates being higher in developing countries.
Rates of malignancy diagnosis ranged from $18.5 \%{ }^{[6]}$ to $100 \%$ in two studies performed in Scotland and India. ${ }^{[4,5]}$ The most common malignancies diagnosed on pleural biopsy in these studies were adenocarcinoma (both primary lung and metastatic disease) and mesothelioma. Other malignancies included lymphomas, anaplastic carcinoma, chondrosarcoma and atypical carcinoid tumours.

There is a paucity of evidence for the use of closed blind pleural biopsy in the South African (SA) setting. Chris Hani Baragwanath Academic Hospital (CHBAH) in Soweto, Johannesburg, is the largest hospital in SA, and indeed in the southern hemisphere, but there have been no studies investigating pleural biopsies in the work-up of exudative pleural effusions at this hospital. 


\section{Objectives}

To determine the aetiology, frequency and change in profile of histopathological diagnoses on pleural biopsy specimens at $\mathrm{CHBAH}$ over the period 1 January 2001 - 31 December 2015.

\section{Methods \\ Study population and data collection}

We conducted a retrospective review of closed blind pleural biopsy specimens taken using an Abrams needle at $\mathrm{CHBAH}$ over the period 1 January 2001 - 31 December 2015. Pleural biopsy specimens taken during this period were submitted to the National Health Laboratory Service (NHLS), where they were analysed by the Department of Anatomical Pathology. Two databases (DISA and TrakCare) were accessed and the reports issued were reviewed. The following information was recorded: age of patient (excluded from study if $<18$ years), gender, HIV status if noted in the report, adequacy of specimen submitted, histopathological diagnosis made and pleural fluid adenosine deaminase (ADA) result, as well as Ziehl-Neelsen (ZN) stain result if noted on the final report.

Patient names and hospital numbers were not collected. Ethics approval was obtained from the University of the Witwatersrand Human Research Ethics Committee (ref. no. 160772).

\section{Statistical analysis}

Data were analysed using Stata version 13.1 (StataCorp, USA). Continuous variables were summarised using means and standard deviations (SDs). Tests for normality were conducted using skewness/ kurtosis testing. Significant associations in contingency tables were assessed using Pearson's $\chi^{2}$ test, and in some cases logistic regression analysis was conducted. Results with $p$-values $<0.05$ were taken as statistically significant.

\section{Results}

A total of 1266 pleural biopsies were reviewed from reports extracted from the NHLS records. Of these, 253 samples were excluded from the study: 222 samples had no patient age specified, 21 were specified with an age $<18$ years, and 10 were submitted from other hospitals. Repeat specimens were submitted for 23 patients: 14 of these samples were inadequate on first sampling, with 5 reflecting an inadequate sample once again on repeat sampling, 4 had a diagnosis of malignancy on the first sample confirmed on the second sample, 3 reflected pleuritis on the first sample confirmed on the second sample, 1 could not be processed on first sampling owing to a laboratory error and therefore a second sample was taken that confirmed pleuritis, and 1 was inconclusive on the first sample, the second sample reflecting primary adenocarcinoma. Samples that had a diagnosis made and confirmed on the second sample were included in the study as a single entry to avoid duplication of diagnoses; however, all inadequate samples and the inconclusive sample were included as distinct entries to allow more accurate assessment of inadequate sampling rates.

A total of 1013 samples were included in the study. Of these, $573(56.6 \%)$ were from males and $440(43.4 \%)$ from females. Of the samples, $780(77.0 \%)$ were considered adequate and $233(23.0 \%)$ were inadequate (Fig. 1). The reasons for samples being considered inadequate for assessment included lack of pleural tissue in the sample, lack of adequate pleural tissue in the sample, and samples damaged to the extent that adequate histological assessment could not be performed. Of the samples considered adequate, 43 were inconclusive following assessment (no specific histopathological diagnosis was made).
Fig. 2 shows the distribution of total samples submitted according to year. The largest number of samples submitted was in 2001 $(n=157)$ and the smallest in $2006(n=33)$. The greatest proportion of inadequate samples was submitted in 2015 (36.7\%), with a trend upwards in the last 5 years (Fig. 3). The majority of specimens submitted had no HIV status specified for the patient; 364 (35.9\% of specimens) had HIV status specified, and of these patients 162 (44.5\%) were HIV-negative and 202 (55.5\%) were HIV-positive.

The histopathological diagnoses made on pleural biopsy in this study were divided into four categories: granulomatous inflammation (necrotising, non-necrotising and undefined granulomatous inflammation), malignancy, nonspecific pleuritis and suppurative pleuritis.

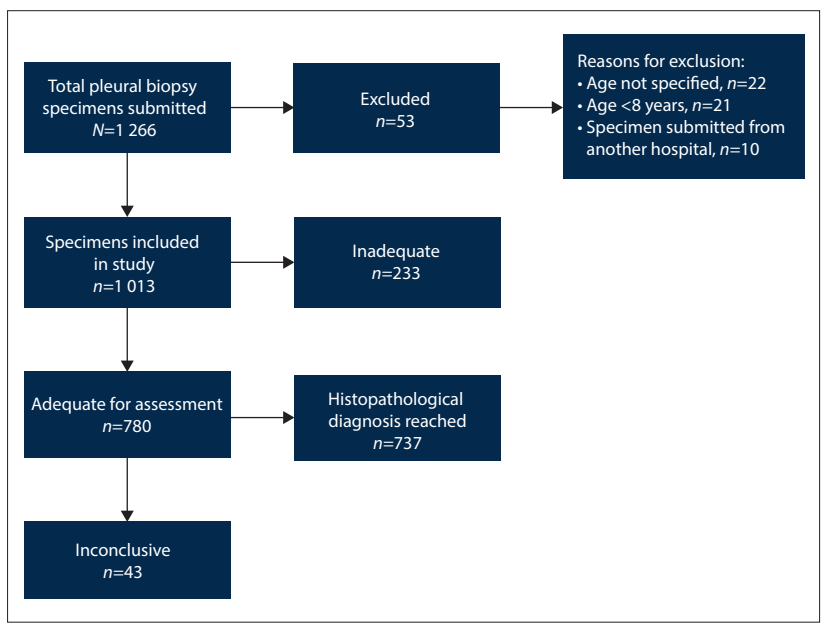

Fig. 1. Study enrolment.

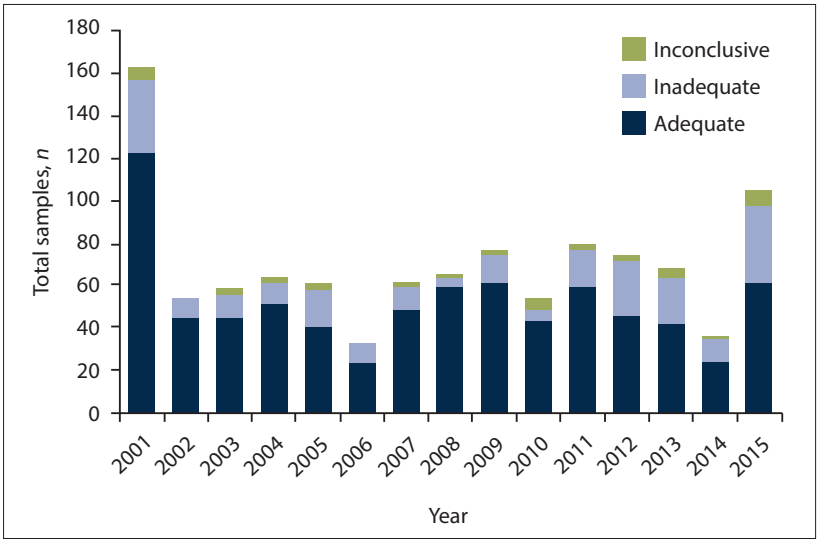

Fig. 2. Sample numbers according to year.

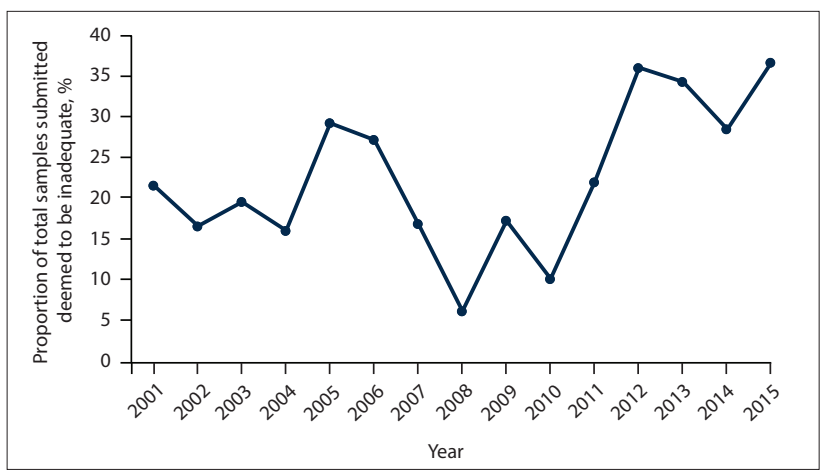

Fig. 3. Distribution of proportion of inadequate samples submitted by year. 


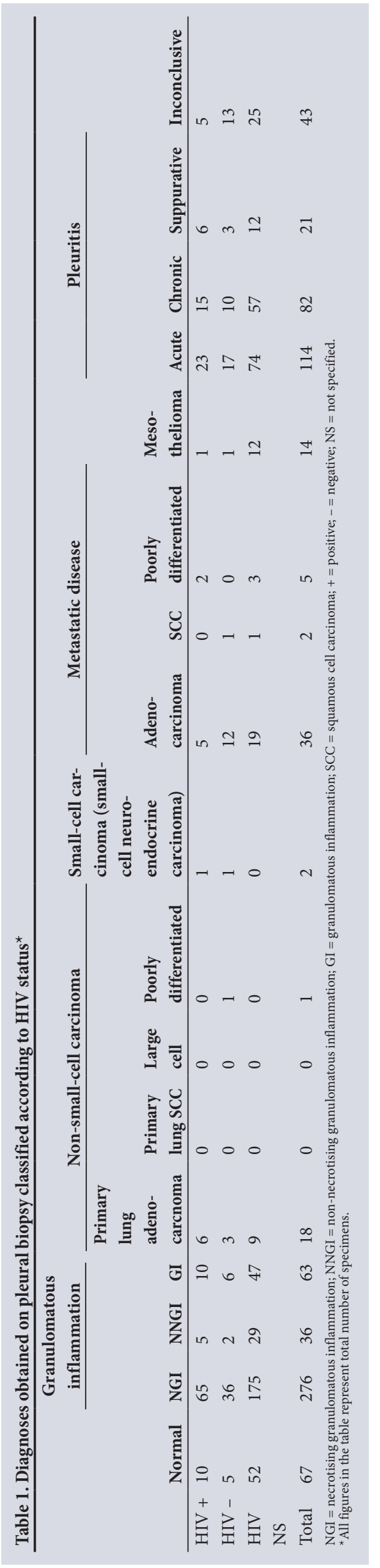

Of the adequate specimens submitted, 67 (8.6\%) were found to be normal pleural tissue on histopathological examination. Granulomatous inflammation was found in 375 of the 780 specimens (48.1\%) (Table 1). Of these, 276 (73.6\%) demonstrated necrotising granulomatous inflammation, $36(9.6 \%)$ non-necrotising granulomatous inflammation and $63(3.75 \%)$ granulomatous inflammation that was not defined as either necrotising or non-necrotising. The mean (SD) age for the diagnosis of granulomatous inflammation was 44 (15) years. There was no significant association between HIV status and any type of granulomatous inflammation ( $p=0.382)$. Only 18 of the granulomatous specimens had an ADA result noted. There was no statistically significant association between any level of $\mathrm{ADA}$ and granulomatous inflammation; however, this may merely reflect the small number of ADA results included.

This study also recorded the ZN stain result for the histopathological specimen, if done. Of note, this stain was only done if the pathologist found features of granulomatous inflammation or, in some cases, pleuritis. However, not all specimens with features of granulomatous inflammation had the stain performed: 298 specimens were stained, and 258 of these had granulomatous inflammation (126 of these were ZN-positive and 132 were $\mathrm{ZN}$-negative). There was also no statistically significant association between a positive $\mathrm{ZN}$ result and necrotising or non-necrotising granulomatous inflammation ( $p=0.793)$.

Of the 780 specimens, 78 (10.0\%) showed malignancy (Table 1). The majority of the malignancies were metastatic adenocarcinomas (46.2\%), with primary lung adenocarcinoma (determined on immunohistochemical staining) second most common (23.1\%), followed by mesothelioma (17.9\%). The mean (SD) age for patients diagnosed with metastatic adenocarcinoma was 58 (14) years, that for primary lung adenocarcinoma 57 (12) years and that for mesothelioma 57 (14) years. Other malignancy diagnoses were 4 cases of adenocarcinoma of unknown primary, 3 cases of squamous cell carcinoma of unknown primary and 1 case of plasmablastic lymphoma. There was no statistically significant variance in age group affected when different malignancy diagnoses were compared with each other. Forty-eight percent of patients diagnosed with malignancy were at least 60 years of age. Logistic regression analysis showed statistically significant increased odds of a cancer diagnosis in the age groups 40 - 49 years (odds ratio (OR) 8.7, $95 \%$ confidence interval (CI) $1.1-66.9$; $p=0.038$ ), 50 - 59 years (OR 12.4, 95\% CI 1.6 - 95.0; $p=0.015$ ) and $\geq 60$ years (OR 23.0,95\% CI $3.1-171.3$; $p=0.002$ ). There was no significant gender difference between malignancy diagnoses $(p=0.450)$. There was also no significant association between HIV status and malignancy diagnosis. Logistic regression analysis demonstrated lower odds for being diagnosed with cancer in samples from patients who were HIV-positive (OR 0.5, 95\% CI $0.2-0.9 ; p=0.040$ ).

In HIV-positive patients, the odds of being diagnosed with a 'non-cancer' diagnosis (including granulomatous inflammation and pleuritis) were higher than in HIV-negative patients (OR 2.16, 95\% CI $1.03-4.51 ; p=0.040$ ).

The diagnosis of pleuritis was made on 217 (27.8\%) of the specimens. Acute pleuritis accounted for $114(52.5 \%)$ of these specimens. Chronic pleuritis was found in 82 specimens $(37.8 \%)$ and suppurative pleuritis in 21 (9.7\%). There was no statistically significant association between the distinct types of pleuritis and HIV status.

\section{Discussion}

Pleural effusions are a common presentation in medical units around the world, and the work-up of a patient presenting with an exudative pleural effusion is extremely important for timely diagnosis and treatment. Blind closed pleural biopsy has been used as a relatively inexpensive means to reach a diagnosis. The majority of pleural biopsies at $\mathrm{CHBAH}$ are done by junior staff (registrars and medical officers) and not by specialist pulmonologists, as is the standard in more resource-rich settings. Despite this, $72.8 \%$ of all samples submitted led to a histopathological diagnosis, suggesting that pleural biopsy remains a valuable tool in our setting. However, the rate of inadequate samples submitted at this hospital averaged $22.6 \%$ per year, with a trend upwards in the years since 2010. This probably reflects inexperience and inadequate training of staff in correct sampling technique, as it compares poorly with rates ranging from $4.2 \%$ to $21 \%$ in other studies. ${ }^{[2,3]}$

Resource-related difficulties experienced at $\mathrm{CHBAH}$ may have impacted on sample numbers in some years. These difficulties included a shortage of, or damaged, Abrams needles. There are no clear retrospective records indicating which years these difficulties may have affected. 
Almost half (48.1\%) of all adequate samples reviewed demonstrated some form of granulomatous inflammation, with the predominant form being necrotising granulomatous inflammation (73.6\%). The presence of granulomas in a histological specimen cannot be considered diagnostic of Mycobacterium tuberculosis, and other causes of granulomatous inflammation should be considered. However, in the developing world, with a high prevalence of $\mathrm{TB}$, the presence of granulomas should raise concerns for $\mathrm{TB}$ as a top differential diagnosis. Of the 780 adequate specimens, 134 (17.2\%) showed positive ZN staining for acid-fast bacilli. However, not all specimens were stained for acid-fast bacilli, including a substantial proportion of specimens that showed granulomatous inflammation. This study also showed that there is no statistically significant association between $\mathrm{ZN}$ positivity and type of granulomatous inflammation (necrotising v. non-necrotising). Other studies in developing countries have shown a TB incidence on pleural biopsy of $32.7-48.9 \%{ }^{[7-9]}$ Despite the higher prevalence of TB in HIV-positive individuals, this study failed to show a significant association between HIV-positive status and granulomatous inflammation $(p=0.382)$. Unfortunately, owing to the small number of samples with HIV status and ZN result included, no meaningful statistics could be derived on the association between HIV status and ZN positivity.

Numerous studies have found an association between high ADA levels and TB. ${ }^{[9-13]}$ The level above which an ADA result should be considered suggestive of $\mathrm{TB}$ has varied across studies, ranging from $30 \mathrm{IU} / \mathrm{L}$ to $70 \mathrm{IU} / \mathrm{L}^{[10,14]}$ In some cases in the present study, ADA results were provided on the report (obtained from previous pleurocentesis specimens submitted). However, a very low number of ADA results were included, and the lack of statistical significance in the relationship between ADA and granulomatous inflammation is therefore probably simply a result of low sample numbers.

Malignancies were diagnosed in $10.0 \%$ of specimens in this study, compared with rates of $18.5-100 \%$ reported in other studies. ${ }^{[1-10,15-21]}$ We found that the majority of malignancies diagnosed were adenocarcinomas, with $46.2 \%$ of all malignancies being metastatic adenocarcinomas and $23.1 \%$ primary lung adenocarcinomas (determined using immunohistochemical staining). Pereyra et al. ${ }^{[21]}$ and Bhattacharya et al. ${ }^{[18]}$ reported rates for adenocarcinoma of $40.3 \%$ and $65 \%$ of all malignancy diagnoses, respectively. Mesothelioma (a primary pleural malignancy) accounted for $17.9 \%$ of malignancies diagnosed in the present study. This is comparable to other studies, which showed rates of $9.4-19 \% \cdot{ }^{[2,7,8]}$

The present study supported the finding by Kalaajieh ${ }^{[7]}$ that the rate of diagnosis of malignancy on pleural biopsy increases with increasing age above 50 years. ${ }^{[7]}$ We showed that there are statistically significantly increased odds of malignancy with older age, with an increasing OR for every decade.

Interestingly, in the present study there was no statistically significant association between HIV status and granulomatous inflammation or malignancy. Despite only $35.9 \%$ of specimens having HIV status noted, logistic regression analysis demonstrated that the odds of being diagnosed with malignancy were higher in the HIV-negative subgroup of patients, and the odds of being diagnosed with a 'non-cancer' diagnosis (including pleuritis and granulomatous inflammation) were significantly greater in the HIVpositive subgroup. The HIV-positive subgroup of patients was younger (mean age 42 years) than the HIV-negative group (mean age 48 years). It may be that the lower prevalence of malignancy in HIV-positive patients in this study simply reflects the fact that fewer HIV-positive than HIV-negative patients survive to the age groups with a higher prevalence of malignancy.
Pleuritis is a nonspecific histopathological finding. In this study, cases of pleuritis were subdivided into acute, chronic and suppurative, and of all the patients, $27.8 \%$ had some form of pleuritis. Of these, only 6 had a positive $\mathrm{ZN}$ result (chronic pleuritis $n=3$, suppurative pleuritis $n=2$, acute pleuritis $n=1)$.

\section{Study limitations}

The major strength of this study is the large sample size compared with other studies. However, our findings must be considered in the light of potential complications. Firstly, the study focused on a retrospective review of laboratory records. Patient records (kept as paper records at this hospital) were not utilised to add more clinical information such as original indication for performing the biopsy, other comorbidities, clinical status of the patient, or other findings that might have provided supportive information for the diagnosis. Secondly, this study relied on reports generated by the Department of Anatomical Pathology at the NHLS. As this was a retrospective review, it did not include second-party validation of the diagnosis proposed on the original report. Samples obtained may have been influenced by the experience level of the clinician performing the procedure, and the histopathological diagnosis may have been influenced by the experience level of the histopathologist assessing the specimen. Finally, no standardised information form was submitted when the original biopsy specimen was sent to the laboratory. Retrospective review of the reports indicated that a substantial proportion of the biopsy specimens submitted had information missing, such as HIV status and ADA result.

\section{Conclusions}

Patients with exudative pleural effusions require work-up to diagnose the cause. This may be elucidated by sputum microscopy, bronchoscopy or cytology. However, we have demonstrated that blind closed pleural biopsy remains a valuable tool in the work-up of these patients in resource-limited settings, particularly in the diagnosis of granulomatous disease and when there is a possibility of malignancy, especially in an older patient. The study also showed this investigation to be valuable in the diagnosis of TB in a setting of high HIV prevalence, especially in developing countries.

The high numbers of inadequate biopsy specimens submitted suggest that further training in sampling technique may improve diagnostic rates.

Declaration. This study was done in fulfilment of the requirements of JRE's MMed in internal medicine with the University of the Witwatersrand, Johannesburg.

Acknowledgements. We thank Mrs Marie Suleman and the staff of the NHLS for their assistance in obtaining the necessary data from a vast database, and Dr G Olorunfemi for his endless patience and assistance with the statistical analysis and interpretation.

Author contributions. JRE and MLW conceived the study. JRE collected the data and prepared the manuscript. MLW and CNM were responsible for critical revision of the manuscript. $\mathrm{MH}$ assisted with access to the NHLS data.

Funding. None.

Conflicts of interest. None.

1. Ngoh HL. Pleural effusion in 100 Malaysian patients. Med J Malaysia 1991;46(4):301-308.

2. Chakrabarti B, Ryland I, Shepard J, Warburton CJ, Earis JE. The role of Abrams percutaneous pleural biopsy in the investigation of exudative pleural effusions. Chest 2006;129(6):1549-1555. https://doi, org/10.1378/chest.129.6.1549 
3. Maskell NA, Gleason FV, Davies RJ. Standard pleural biopsy versus CT-guided cutting-needle biopsy for diagnosis of malignant disease in pleural effusions: A randomized controlled trial. Lancet 2003;361(9366):1326-1331. https://doi.org/10.1016/S0140-6736(03)13079-6

4. McLean AN, Bicknell SR, McAlpine LG, Peacock AJ. Investigation of pleural effusion: An evaluation of the new Olympus LTF semiflexible thoracofiberscope and comparison with Abram's needle biopsy. Chest 1998;114(1):150-153. https://doi.org/10.1378/chest.114.1.150

. Manju R, Venkateswara Babu R, Vinod Kumar S, Badhe AS. A comparative study by using Abrams and Cope needles in pleural biopsy exudative effusions. World J Med Sci 2012;7(3):151-154.

6. Al-Shimemeri AA, Al-Ghadeer HM, Giridhar HR. Diagnostic yield of closed pleural biopsy in exudative pleural effusion. Saudi Med J 2003;24(3):282-286.

Kalaajieh WK. Etiology of exudative pleural effusions in adults in North Lebanon. Can Respir J 2001;8(2):93-97. https://doi.org/10.1155/2001/652418

8. Zuberi FF, Zuberi BF, Ali SK, Hussain S, Mumtaz F. Yield of closed pleural biopsy and cytology in exudative pleural effusion. Pak J Med Sci 2016;32(2):356-360.

9. Solooki M, Miri M. Approach to undiagnosed exudative pleural effusion: The diagnostic yield of blind pleural biopsy. Caspian J Intern Med 2013;4(2):642-647.

10. Verma SK, Dubey AL, Singh PA, Tewerson SL, Sharma D. Adenosine deaminase (ADA) level in tubercular pleural effusion. Lung India 2008;25(3):109-110.

11. Reechaipichitkul W, Kawamatawong T, Teerajetgul Y, Patjanasoontorn B. Diagnostic role of pleural fluid adenosine deaminase in tuberculous pleural effusion. Southeast Asian J Trop Med Public Health 2001;32(2):383-389.

12. Valdés L, Alvarez D, San José E, et al. Value of adenosine deaminase in the diagnosis of tuberculous pleural effusions in young patients in a region of high prevalence of tuberculosis. Thorax 1995;50(6):600-603. https://doi.org/10.1136/thx.50.6.600

13. Chen ML, Yu WC, Lam CW, Au KM, Kong FY, Chan AY. Diagnostic value of pleural fluid adenosine deaminase activity in tuberculous pleurisy. Clin Chim Acta 2004;341(1-2):101-107. https://doi. org/10.1016/j.cccn.2003.11.016
14. Banales JL, Pineda PR, Fitzgerald JM, Rubio H, Selman M, Salazar-Lezama M. Adenosine deaminase in the diagnosis of tuberculous pleural effusions: A report of 218 patients and review of the literature. in the diagnosis of tuberculous pleural effusions: A report of 218 part
Chest 1991;99(2):355-357. https://doi.org/10.1378/chest.99.2.355

15. Mungall IP, Cowen PN, Cooke NT, Roach TC, Cooke N. Multiple pleural biopsy with the Abrams needle. Thorax 1980;35(8):600-602. https://doi.org/10.1136/thx.35.8.600

16. Edmondstone WM. Investigation of pleural effusion: Comparison between fibreoptic thoracoscopy needle biopsy and cytology. Respir Med 1990;84(1):23-26. https://doi.org/10.1016/S0954$6111(08) 80089-3$

17. Prakash UB, Reiman HM. Comparison of needle biopsy with cytologic analysis for the evaluation of pleural effusion: Analysis of 414 cases. Mayo Clin Proc 1985;60(3):158-164. https://doi.org/10.1016 S0025-6196(12)60212-2

18. Bhattacharya S, Bairagya TD, Das A, Mandal A, Das SK. Closed pleural biopsy is still useful in the evaluation of malignant pleural effusion. I Lab Physicians 2012:4(1):35-38. https://doi. org/10.4103/0974-2727.98669

1. Nance KV Shermer RW, Askin FB. Diagnostic efficacy of pleural biopsy as compared with that of pleural fluid examination. Mod Pathol 1991;4(3):320-324.

20. Salyer WR, Eggleston JC, Erosan YS. Efficacy of pleural needle biopsy and pleural fluid cytopathology in the diagnosis of malignant neoplasm involving the pleura. Chest 1975;67(5):536-539. https://doi. org/10.1378/chest.67.5.536

21. Pereya MF, San-José E, Ferreiro L, et al. Role of blind closed pleural biopsy in the management of pleural exudates. Can Respir J 2013;20(5):362-366. https://doi.org/10.1155/2013/731352

Accepted 7 June 2018. 